\title{
Energy Efficient Wireless Networks
}

\author{
Yujin Lim $(\mathbb{D}),{ }^{1}$ Gianluigi Ferrari $(\mathbb{D}),{ }^{2}$ Hideyuki Takahashi $\mathbb{D}^{3},{ }^{3}$ and Rossana M. C. Andrade ${ }^{4}$ \\ ${ }^{1}$ Sookmyung Women's University, Seoul, Republic of Korea \\ ${ }^{2}$ University of Parma, Parma, Italy \\ ${ }^{3}$ Tohoku Gakuin University, Sendai, Japan \\ ${ }^{4}$ Federal University of Ceará, Fortaleza, Brazil
}

Correspondence should be addressed to Yujin Lim; yujin91@sookmyung.ac.kr

Received 30 May 2019; Accepted 30 May 2019; Published 12 June 2019

Copyright (C) 2019 Yujin Lim et al. This is an open access article distributed under the Creative Commons Attribution License, which permits unrestricted use, distribution, and reproduction in any medium, provided the original work is properly cited.

As IoT services become vitalized, this leads to an explosion of sensor nodes more and more. As the energy consumption of a wireless network increases with the number of nodes, it becomes critical to reduce the energy consumption of a wireless network. The sensor nodes are required to function for from a few months to a few years without recharging once they are installed. In order to enlarge the lifetime of the nodes, an energy efficient scheme is needed to save the energy of the nodes. Now, the energy efficiency has become one of the key criteria for designing wireless networks, not only because of the environmental concerns, but also practical reasons of the nodes in wireless networks. Even though a significant number of studies taking evolutionary approaches are in progress to give advancements of the energy efficient wireless networks, much effort is still required to crystallize the energy efficient wireless networks.

The scope of this special issue is in line with recent contributions from academia and industry to tackle the technical challenges in order to concretize the energy efficient wireless networks. For the current issue, we are pleased to introduce a collection of papers covering a range of topics as follows:

(i) Switching strategy for energy efficient wireless networks

(ii) Clustering protocol for energy efficient wireless networks

(iii) Transmission power control for energy efficient wireless networks (iv) Vertical handover algorithm for energy efficient wireless networks

(v) Optimization of directional antennas for energy efficient wireless networks

As always, we appreciate the high-quality submissions from authors and the support of the community of reviewers.

\section{Conflicts of Interest}

The guest editors declare no conflicts of interest.

Yujin Lim

Gianluigi Ferrari

Hideyuki Takahashi

Rossana M. C. Andrade 


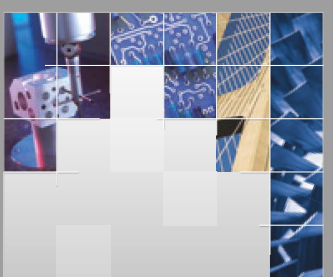

\section{Enfincering}
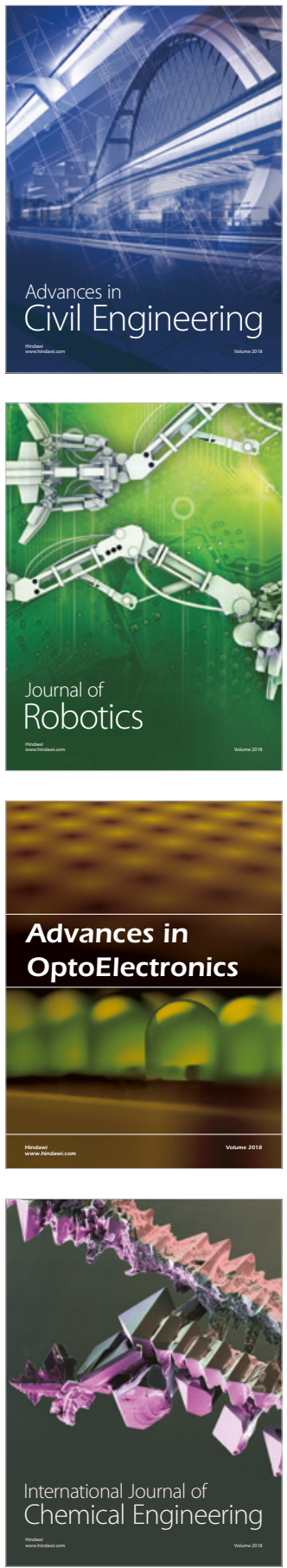

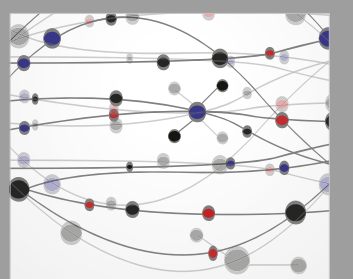

\section{Rotating \\ Machinery}

The Scientific World Journal

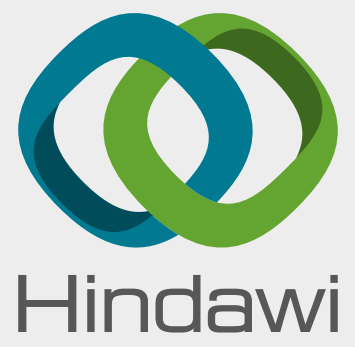

Submit your manuscripts at

www.hindawi.com
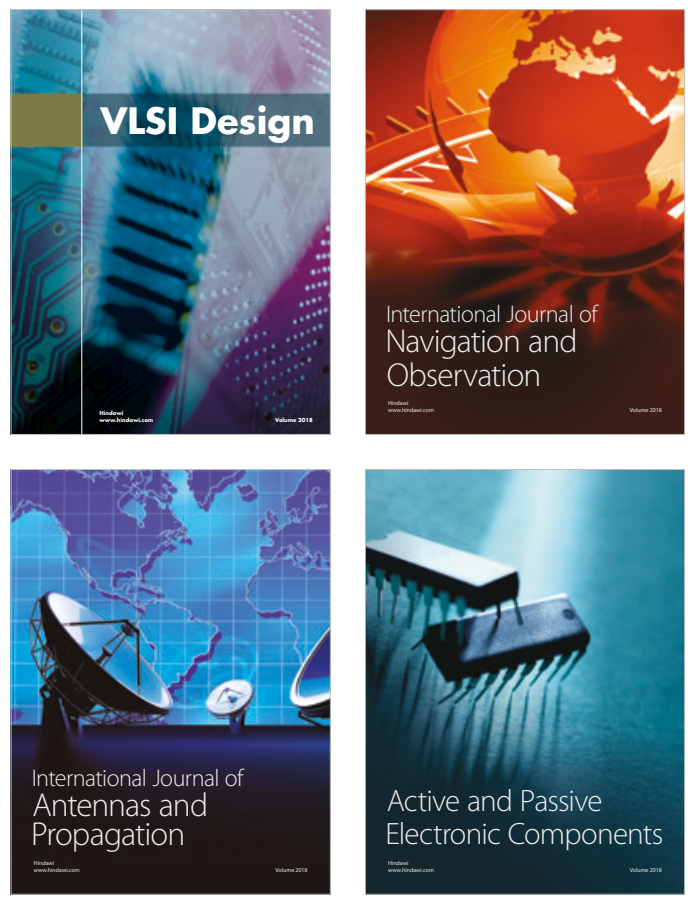
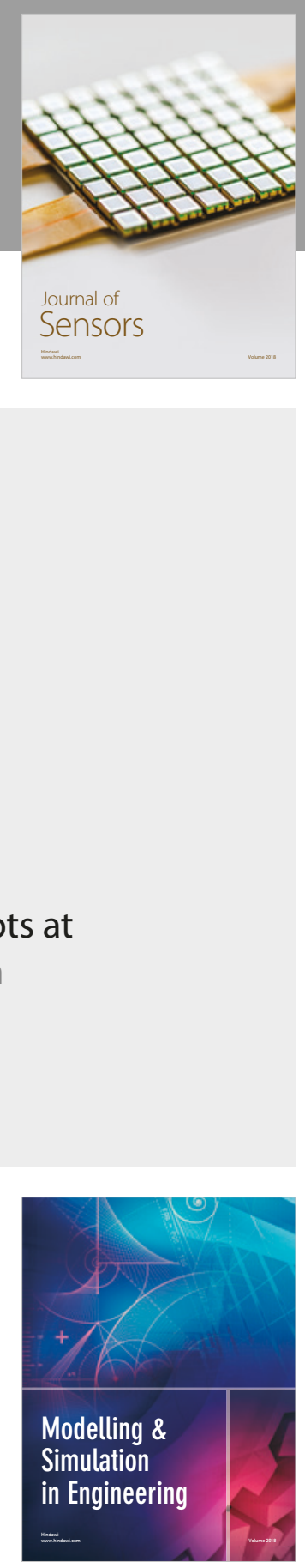

\section{Advances \\ Multimedia}
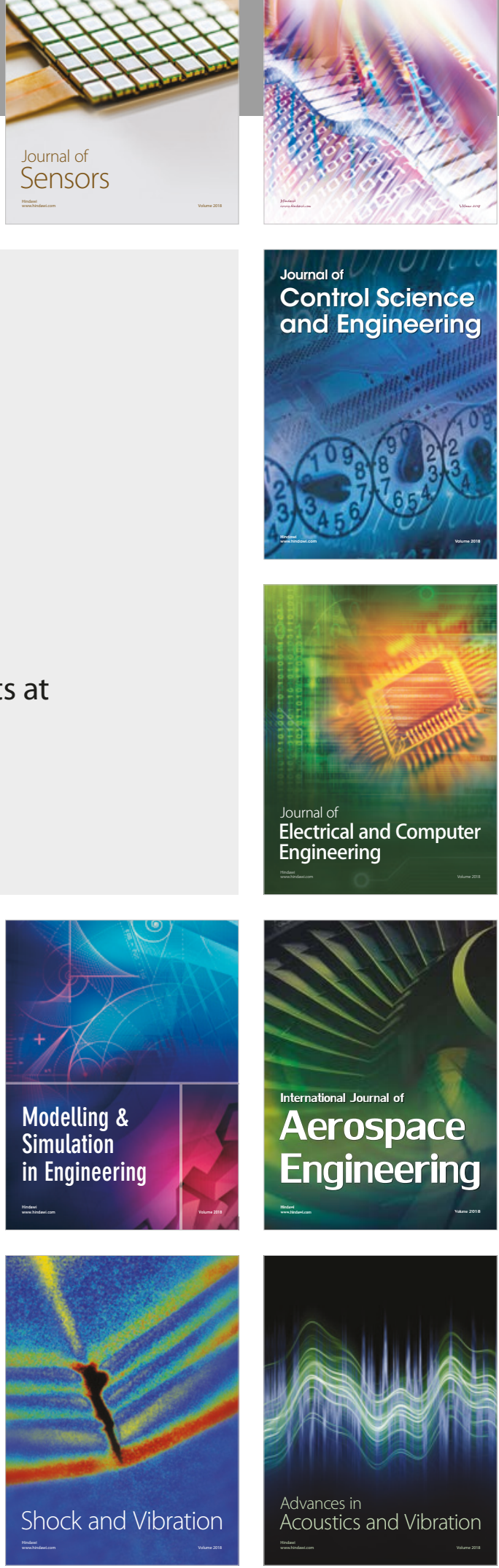